\title{
Syntheses of MTT-Type Zeolites with the Presence of Both Octyltrimethylammonium Chloride and SFE-Type Zeolite Seeds
}

\author{
Duozheng Ma, Wenhua Fu, Chuang Liu, Jun Liang, Zhiqing Yuan, Weichuan Tao, Zhendong Wang,* \\ and Weimin Yang*
}

Cite This: ACS Omega 2021, 6, 23598-23606

Read Online

ABSTRACT: Structure-directing agents (SDAs) play important roles in directing the formation of specific zeolite frameworks. Mechanisms and working hypothesis were proposed for understanding how SDAs work during the crystallization of zeolites. The lately reported cooperative structure-directing effect based on the investigation into the synthetic system containing both seed and organic species is one of them and is believed to be effective for synthesizing zeolites which are difficult to access or with novel structures. However, more examples are still needed to support the thesis. Herein, we report for the first time the syntheses of MTTtype zeolites with the simultaneous presences of octyltrimethy-

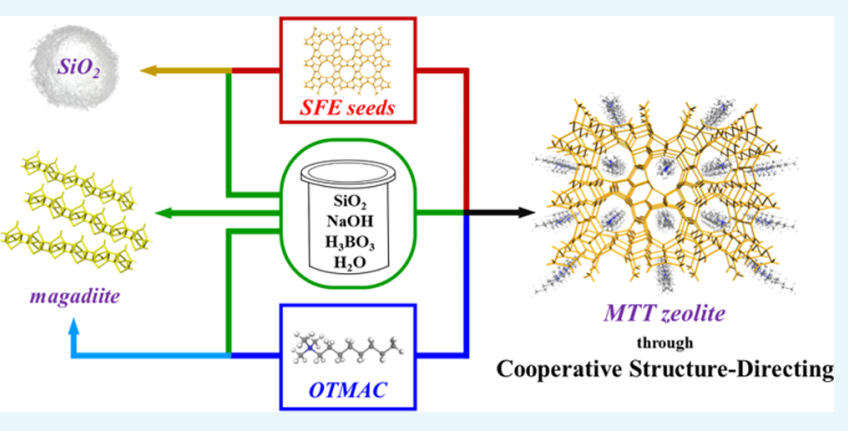
lammonium chloride (OTMAC) and SFE zeolite seeds in the borosilicate system. SFE borosilicate zeolites serve as exotic seeds for the crystallization of MTT-type zeolites and together with OTMAC play cooperative structure-directing roles. Besides, Al,B-MTT and heteroatom (Zr, V, and Fe)-incorporated MTT-type zeolites were directly synthesized with the introduction of metal sources into the borosilicate system. Physicochemical properties of the obtained MTT zeolites were characterized by X-ray diffraction, scanning electron microscopy, transmission electron microscopy, $\mathrm{N}_{2}$ physisorption, $\mathrm{NH}_{3}-\mathrm{TPD},{ }^{27} \mathrm{Al} \mathrm{NMR},{ }^{13} \mathrm{C}$ NMR, ${ }^{11} \mathrm{~B} \mathrm{NMR}, \mathrm{UV}-$ visible spectroscopy, and UV Raman spectroscopy. The hereinreported phenomenon provides an example to better understand the mechanism of zeolite crystallization, and the synthesized zeolites may act as promising catalytic materials in several organic reactions.

\section{INTRODUCTION}

Zeolites are an important class of crystalline microporous materials with industrial applications in ion exchange, adsorption, and catalysis. ${ }^{1}$ The syntheses of zeolites usually need the use of $\mathrm{N}$-containing amines or ammoniums which are commonly called organic structure-directing agents (OSDAs), acting as (1) pore fillers, (2) structure-directing agents (SDAs), and (3) templates. The introduction of OSDAs has favored the syntheses of high-silica zeolites and promoted the discovery of new zeolite structures. $^{2-4}$ However, OSDA increases the cost of zeolite synthesis and results in energy consumption and pollution during its removal via calcination. ${ }^{3,5-7}$ The seed-directed synthesis (SDS) routes of zeolites have made great progress recently because they avoided or decreased the use of OSDAs. In OSDA-free syntheses of zeolites, zeolite seeds actually direct the crystallization and thus are considered as the third SDAs. ${ }^{8}$ According to the structural similarity between the zeolite seed and product, the SDS routes could be divided into conventional seeds and exotic seeds. Conventional seeds mean that the seeds and products are of the same structure, while exotic seeds mean that the structure of the product is different from that of the seeds. ${ }^{8}$ There are a lot of zeolites that have been synthesized through SDS routes, including beta (*BEA), ${ }^{9,10}$ ZSM-12 (MTW), ${ }^{11}$ EU-I (EUO), ${ }^{7}$ ZJM-2 (FER), ${ }^{12}$ levyne (LEV), ${ }^{13}$ mordenite (MOR), ${ }^{14}$ ZSM-5 (MFI), and ZSM-11 (MEL). ${ }^{15}$ When used as "seed", the amount of zeolite in the starting mixture was usually less than $20 \%$ corresponding to the amount of silica source. ${ }^{16}$ Besides, using zeolites including FAU, ${ }^{17-19}$ GIS, ${ }^{20,21} * \mathbf{B E A}^{22}$ and $\mathbf{L T L}^{23}$ as silica and/or aluminum source in the starting mixture is another strategy for preparing target zeolites, which results in both the "green" syntheses of known zeolites ${ }^{10}$ and the discovery of novel zeolite. ${ }^{24}$

Lately, the cooperative structure-directing method was reported. ${ }^{25}$ Hydrothermally stable aluminogermanosilicate IWR zeolite was synthesized with the simultaneous presences of both *BEA seed and choline, which together direct the crystallization of IWR zeolite. Fang et al. synthesized NU-87 (NES) zeolite by adding zeolite EU-1 (EUO) as the exotic

Received: July 17, 2021

Accepted: August 24, 2021

Published: September 2, 2021 
seed in the presence of decamethonium bromide. ${ }^{26}$ Such syntheses required the presences of both an exotic zeolite and an organic compound, and they directed the formation of the product together. It is a promising route for the syntheses of zeolites which have not been accessible by traditional hydrothermal synthetic methods, as well as zeolites with novel structures. However, there is only rather limited examples of such methodology.

The MTT zeolite, first reported as ZSM-23, possesses noninterconnected 10-MR one-dimensional channels with drop-shaped pore openings of $0.52 \times 0.45 \mathrm{~nm}$ in diameter. ZSM-23 showed promising applications in the isomerization of butene and catalytic cracking reactions. ${ }^{27-30}$ Numerous OSDAs have been employed in the synthesis of MTT zeolite, including $N, N$-dimethylformamide, ${ }^{5,31}$ isopropylamine (IPA), ${ }^{28,31}$ ethylene glycol, $^{31}$ and pyrrolidine (PY). ${ }^{32,33}$ Moreover, Moini et al. have used a variety of linear-type diquaternary ammonium, including Me6-diquat-5, Me6diquat-6, Me6-diquat-11, and Me6-diquat-12, to prepare ZSM-23 zeolite. ${ }^{34}$ Studies on the preparations of zeolites using quaternary ammoniums with carbon chain lengths greater than 6 are rather limited, the reason of which may lie in the weak structure-directing ability of this family of compounds. ${ }^{35} \mathrm{Wu}$ et al. prepared Ti-MWW zeolite using octyltrimethylammonium hydroxide $(\mathrm{OTMAOH})$ as OSDA. ${ }^{33}$ However, there is no report on the use of octyltrimethylammonium chloride (OTMAC) to synthesize MTT zeolite in the literature. The broom-like shape of the octyltrimethylammonium molecule matches the one-dimensional straight channel of MTT, inspiring us that it can be utilized to direct the formation of MTT zeolite.

Herein, we report the syntheses of borosilicate MTT-type zeolites with the presence of both linear-type organic species OTMAC and SFE-type zeolite seeds, which act together as the cooperative SDAs. In addition, $\mathrm{Al}, \mathrm{Zr}, \mathrm{V}$, and $\mathrm{Fe}$ atomincorporated MTT zeolites were also successfully synthesized.

\section{RESULTS AND DISCUSSION}

B-SFE zeolite seed (Figures S1 and S2) and OTMAC were used for the syntheses of MTT zeolites. Figure 1 shows the Xray diffraction (XRD) patterns of solid products obtained under different conditions. The XRD pattern of the obtained MTT borosilicate zeolite [run 7 (corresponding to the run number listed in Table S1, indicating the crystallization parameters for the synthesis of the corresponding zeolite)] with the presence of both OTMAC and SFE zeolite seed (Figure 1a) showed a series of characteristic peaks corresponding to the MTT structure, ${ }^{28,32,36,37}$ which are consistent with the peaks of the simulated XRD pattern, proving the successful synthesis of MTT zeolite.

However, when crystallizations were carried out with the presence of only SFE zeolite seed (run 2-4) or OTMAC (run 5-6), only dense-phase $\mathrm{SiO}_{2}$ (Figure $1 \mathrm{~b}$ ) or magadiite (Figure 1c) was obtained. Besides, still no MTT zeolite was obtained by increasing the amount of SFE zeolite seed (run 4) or OTMAC (run 6). The attempt to synthesize zeolite without OTMAC and seed resulted in the formation of magadiite (Figure 1d, run 1). It is worth noting that the addition of OTMAC did not change the synthetic product in comparision with the gel without organics and seeds. This may be due to the fact that OTMAC, as a linear quaternary ammonium, has a relatively weak structure-directing ability for the MTT zeolite structure.

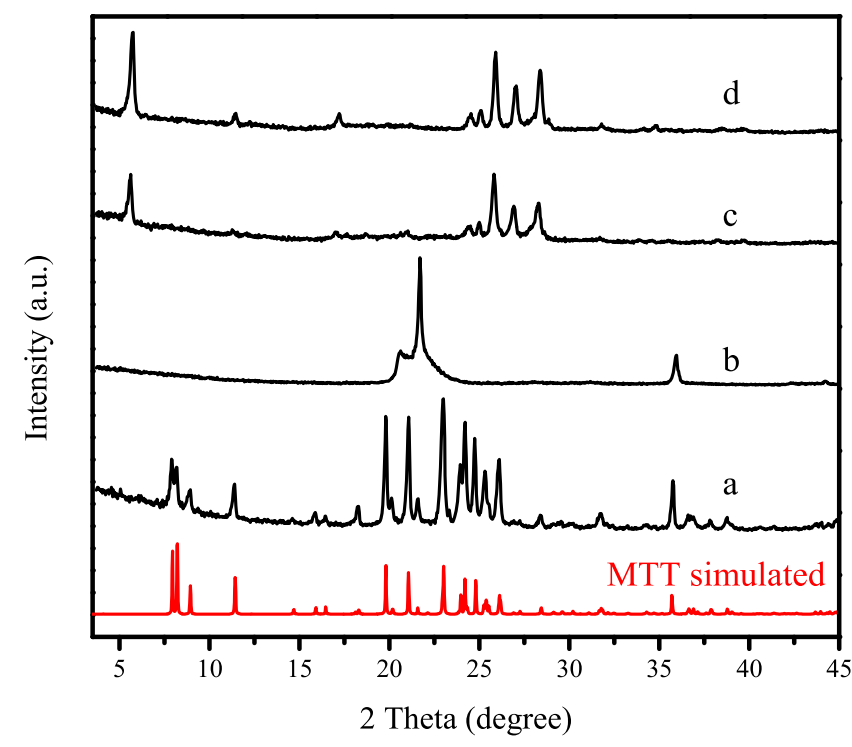

Figure 1. XRD patterns of the obtained products synthesized with the presence of both B-SFE seed and OTMAC, run 7 (a), B-SFE seed, run 3 (b), OTMAC, run 5 (c), and without zeolite seed and OTMAC, run $1(\mathrm{~d})$.

It seems that the SFE zeolite seed and OTMAC together direct the crystallization of MTT zeolite in the herein-adopted system. The seed-directed synthesis without OTMAC in this work falls in the category of SDS without OSDA, in which a working hypothesis was proposed by Okubo et al. ${ }^{16}$ According to the hypothesis, SFE zeolite is a potential seed candidate for the synthesis of MTT zeolite, since they share the same common composite building units ( $\mathrm{jbw}, \mathrm{mtt}$, bik, and ton). Considering that the hypothesis was proposed on the basis of the results of OSDA-free synthesis of beta, mordenite, and ferrierite in the aluminosilicate system, the most likely explanation for the herein-reported phenomena is that the synthesis of MTT borosilicate zeolite follows another mechanism. Both OTMAC and SFE zeolite seed are needed for the synthesis of MTT zeolite, which is similar to the synthesis of IWR zeolite with the presence of both *BEA zeolite seed and choline. ${ }^{25}$ Thus, it is appropriate to say that the synthesis was realized successfully through a cooperative structure-directing method.

Effects of synthetic parameters on the crystallization of MTT zeolites were investigated using starting mixtures with different molar ratios (Table S1). MTT borosilicate zeolites were synthesized with $\mathrm{SiO}_{2} / \mathrm{B}_{2} \mathrm{O}_{3}$ molar ratios ranging from 10 to 80 (run 7-9 in Table $\mathrm{S} 1$ ). The amorphous phase was obtained when the starting $\mathrm{SiO}_{2} / \mathrm{B}_{2} \mathrm{O}_{3}$ molar ratio was lower than 10 . When the initial gel did not contain any boron, in other words, $\mathrm{SiO}_{2} / \mathrm{B}_{2} \mathrm{O}_{3}=$ inf, layered phase searlesite appeared as the only crystalline product (run 10). The XRD patterns of products synthesized under different alkali contents are depicted in Figure $\mathrm{S} 3$. When the $\mathrm{NaOH} / \mathrm{SiO}_{2}$ molar ratios varied from 0.30 to 0.50 , ZSM-48 (*MRE) zeolite (run 11-12) and ZSM12 (MTW) zeolite (run 13) were obtained. The suitable $\mathrm{NaOH} / \mathrm{SiO}_{2}$ molar ratios for the syntheses of MTT zeolites were between 0.60 and 0.65 (run 7 and run 14). Further increasing the $\mathrm{NaOH} / \mathrm{SiO}_{2}$ molar ratio to 0.7 would result in the formation of kenyaite (Figure S3f, run 15). It is worth noting that the successful syntheses of both *MRE and MTW 

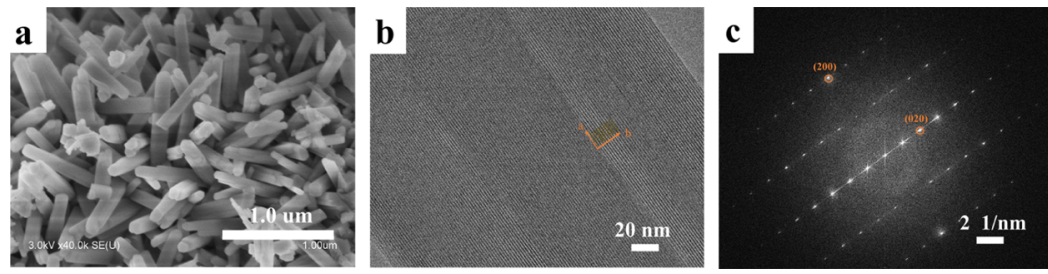

Figure 2. FE-SEM image of B-MTT (a) and its HRTEM pattern (b) and FFT pattern (c).

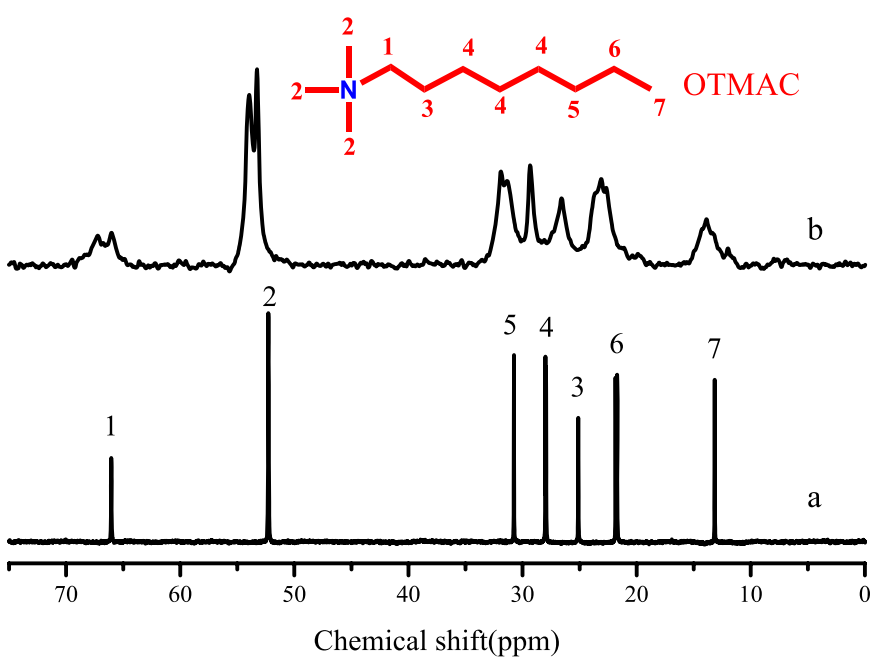

(A)

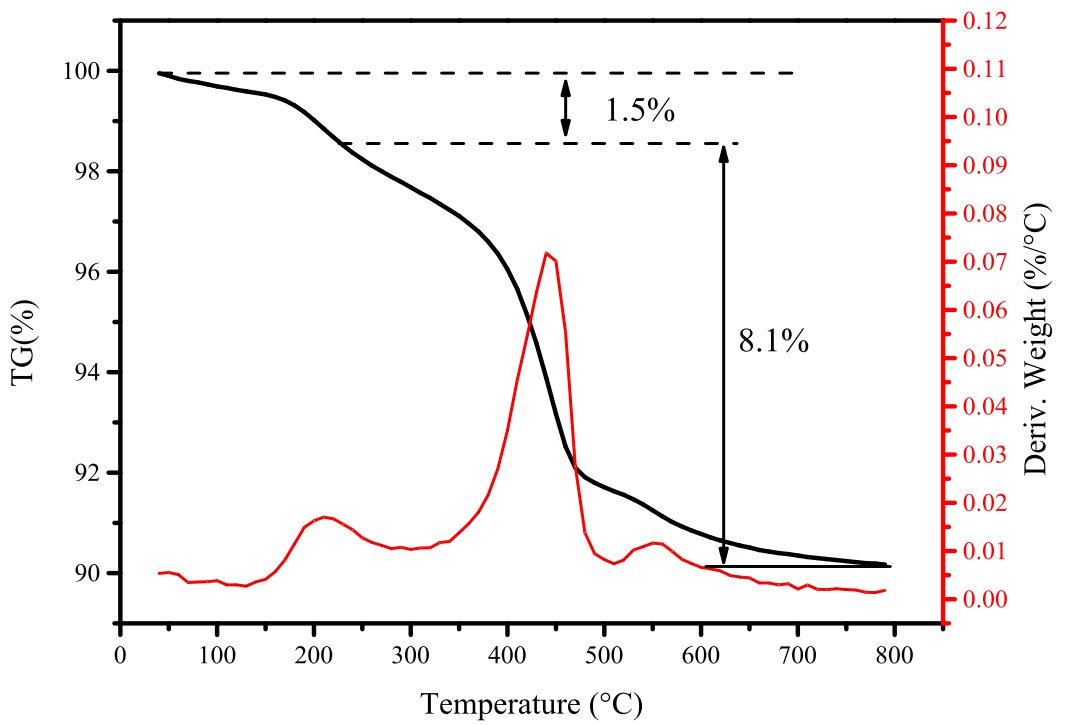

(B)

Figure 3. (A) ${ }^{13} \mathrm{C}$ liquid NMR spectrum of OTMAC in $\mathrm{D}_{2} \mathrm{O}$ (a) and ${ }^{13} \mathrm{C}$ CPMAS NMR spectrum of the as-made B-MTT zeolite, run 7 (b). (B) TG-DTG curve of the as-made B-MTT zeolite, run 7.

zeolites, besides MTT zeolite, followed the cooperative structure-directing effect of exotic SFE seeds and OTMAC.

Water content in the initial gel also possessed great influence on the phases of products. MTT zeolite could be synthesized with $\mathrm{H}_{2} \mathrm{O} / \mathrm{SiO}_{2}$ molar ratios ranging from 20 to 30, while MTW and MFI phases were obtained by increasing water content (run 16-17).

Figure 2 shows the field emission scanning electron microscopy (FE-SEM) image and its high-resolution trans- mission electron microscopy (HRTEM) pattern and the fast Fourier transform (FFT) pattern of B-MTT (run 7, Table S1). The synthesized MTT borosilicate zeolite shows a rodlike morphology with a length of $1000 \mathrm{~nm}$ and a diameter of about $100 \mathrm{~nm}$ (Figure 2a), which is in good agreement with those of ZSM-23 zeolites reported previously. ${ }^{28,29,36}$ The HRTEM image and corresponding FFT pattern (Figure 2b,c) illustrated that the obtained B-MTT zeolite is of high crystallinity and the longest dimension of the crystal is along the direction of $1 \mathrm{D}$ 
channel. The solid yield of MTT zeolite was about 36\% due to the high alkalinity which increased the solubility of silicon and the boron source. ${ }^{38}$ The bulk molar compositions of B-MTT zeolites were much higher than those in the starting gels (run 7-9 in Table S1) because boron species are difficult to be incorporated into the framework. ${ }^{39}$

OTMAC acted as the co-SDA in the synthesis of MTT borosilicate zeolite. The ${ }^{13} \mathrm{C}$ MAS NMR spectrum of the assynthesized MTT borosilicate zeolite (Figure 3A(b), run 7) fits well with the ${ }^{13} \mathrm{C}$ liquid NMR spectrum of OTMAC in $\mathrm{D}_{2} \mathrm{O}$ (Figure 3A(a)), indicating that OTMAC was occluded in the synthesized MTT zeolite without decomposition. The broadness of the resonances in the range of 10-35 ppm was observed because the methyl carbon $\left(-\mathrm{CH}_{3}\right)$ and methylene carbons $\left(-\mathrm{CH}_{2}-\right)$ were in a similar environment, while the splits of the signals ranging from 50 to $70 \mathrm{ppm}$ corresponding to the methyl carbons $\left(-\mathrm{CH}_{3}\right)$ and methylene carbon near the $\mathrm{N}$ atom $\left(-\mathrm{CH}_{2}-\right)$ were caused by the geometric constrain and van der Waals interactions. ${ }^{35}$ Figure $3 \mathrm{~B}$ shows the TG-DTG profiles of the as-made B-MTT (run 7) and confirmed the presence of OTMAC. Several exothermic processes accompanied by continuous weight loss originating from the removal of water and organic species were observed. The weight loss (1.5 wt \%) in the low-temperature region $\left(25-200{ }^{\circ} \mathrm{C}\right)$ was mainly due to the desorption of physically adsorbed water. The weight loss $(8.1 \mathrm{wt} \%)$ in the high-temperature region (200$750{ }^{\circ} \mathrm{C}$ ) was originated from the removal of the occluded organic species.

Samples at different crystallization times were collected and investigated by X-ray diffraction (XRD) and scanning electron microscopy (SEM) to illustrate the crystallization process. At the very beginning of crystallization, diffraction peaks corresponding to SFE zeolite seed were detected (Figure 4,

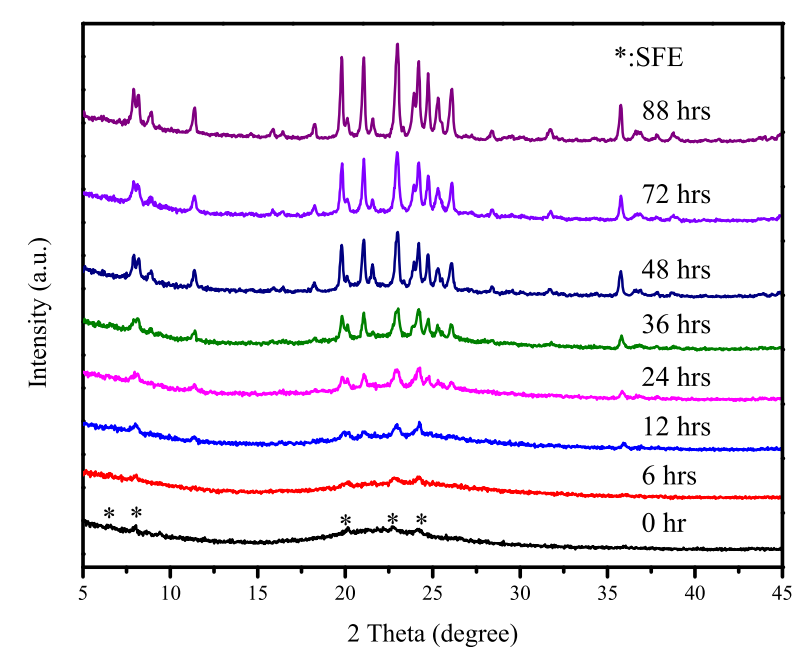

Figure 4. XRD patterns of samples obtained at different crystallization times.

$0 \mathrm{~h})$. The MTT structure was detected after $12 \mathrm{~h}$ crystallization. With prolonging the crystallization time, increases in the intensities of diffraction peaks originating from MTT zeolite were observed (Figure 4, 36-72 h). The fully crystallized MTT zeolite was obtained at $88 \mathrm{~h}$. SEM images of samples obtained during crystallization of the sample run 7 are shown in Figure 5. At 6 h, the solid product shows a wormhole-like morphology (Figure 5a). After crystallization for $12 \mathrm{~h}$, spheres with different sizes are observed (Figure $5 \mathrm{~b}$ ).
After $36 \mathrm{~h}$ of hydrothermal treatment, both spheres and rods are observed. Considering the final rodlike morphology of the B-MTT zeolite and the partially crystalline nature of the sample collected at this stage, the spheres are believed to be amorphous, while the rods are B-MTT zeolite crystals (Figure 5c). With prolonging the crystallization time to $88 \mathrm{~h}$, highly crystalline rod-shaped crystals are obtained (Figure $5 \mathrm{~d}$ ). The size of the MTT zeolite product is significantly larger than that of the SFE seed (Figure S2). ${ }^{39}$

Al,B-MTT zeolites were also directly synthesized with the presences of both SFE zeolite seeds and OCTMA (Figure S4). The morphologies of Al,B-MTT are all rodlike and similar to that of B-MTT. It is worth noting that the introduction of $\mathrm{Al}$ decreased the crystal size of MTT zeolites (Figure S5). The $\mathrm{SiO}_{2} / \mathrm{Al}_{2} \mathrm{O}_{3}$ molar ratios of the solid Al,B-MTT products are lower than those in the gels, which may be caused by the high alkalinity (Table 1). The solid yield of Al,B-MTT zeolite is slightly higher than that of B-MTT because of the introduction of $\mathrm{Al}$ (Table S1). Figure 6 shows the nitrogen adsorptiondesorption isotherms of the Al,B-MTT (run 18, 19, and 20) and B-MTT (run 7) zeolites, and the corresponding textural properties are listed in Table 1 . All isotherms were classified as type I corresponding to the classic microporous material. The textural parameters of Al,B-MTT zeolites, including the total BET area, micropore surface area, external surface area, micropore volume, and total pore volume, are all obviously larger than those of B-MTT (Table 1). It was reported that parts of boron elements would be extracted from the framework during the calcination process. These expelled species may block the pores, leading to the reduced BET area and pore volume. ${ }^{2,40}$ The textural properties of obtained Al,BMTT-type zeolites increase first and then decrease with the increase in $\mathrm{SiO}_{2} / \mathrm{Al}_{2} \mathrm{O}_{3}$ molar ratios (Table 1). The BET area and micropore volume of $\mathrm{Al}, \mathrm{B}-\mathrm{MTT}$ are in good agreement with those of ZSM-23 zeolites synthesized using PY and/or IPA as SDAs. ${ }^{28,32,41}$

The incorporation of $\mathrm{B}$ and $\mathrm{Al}$ was confirmed by solid-state ${ }^{11} \mathrm{~B}$ and ${ }^{27} \mathrm{Al}$ MAS NMR measurements. As shown in Figure $7 \mathrm{~A}$, the narrowly clustered resonances in the range of -5 to 0 ppm are assigned to tetrahedrally coordinated boron species $(\mathrm{B}(4)) .^{40,42,43}$ The presence of a residual line at $-2.4 \mathrm{ppm}$ for B-MTT indicated the existence of a small amount of boron species in the form of twisted $\mathrm{B}(4))^{44}$ The only resonances centered at $\delta=59 \mathrm{ppm}$ in the ${ }^{27} \mathrm{Al}$ MAS NMR spectra of the Al-MTT zeolites with $\mathrm{SiO}_{2} / \mathrm{Al}_{2} \mathrm{O}_{3}$ molar ratios of 200 and 560 (Figure $7 \mathrm{~B}$ ) revealed the incorporation of $\mathrm{Al}$ into the framework and the absence of octahedrally coordinated nonframework $\mathrm{Al}$ species.

Acidic properties of $\mathrm{H}-\mathrm{B}-\mathrm{MTT}$ and $\mathrm{H}-\mathrm{Al}, \mathrm{B}-\mathrm{MTT}$ zeolites with different $\mathrm{SiO}_{2} / \mathrm{Al}_{2} \mathrm{O}_{3}$ molar ratios were investigated by $\mathrm{NH}_{3}$-TPD (Figure 8). A desorption peak at around 125-225 ${ }^{\circ} \mathrm{C}$ was observed in the $\mathrm{NH}_{3}$-TPD curves of B-MTT. Besides, a very small adsorption peak in the high-temperature zone was also observed (Figure 8a). H-Al,B-MTT zeolites possess both weak and strong acid sites. Two peaks corresponding to ammonia desorption from weak acid (centered at 100-250 ${ }^{\circ} \mathrm{C}$ ) and strong acid $\left(250-550{ }^{\circ} \mathrm{C}\right.$ ) were observed (Figure $8 \mathrm{~b}-\mathrm{d})$. The estimation of the acidic sites of various samples is presented in Table S2. The weak acid amount, strong acid amount, and total acid amount of Al,B-MTT vary with the $\mathrm{Al}$ content. More $\mathrm{Al}$ atoms endow $\mathrm{Al}, \mathrm{B}-\mathrm{MTT}$ zeolites with more acid sites. 

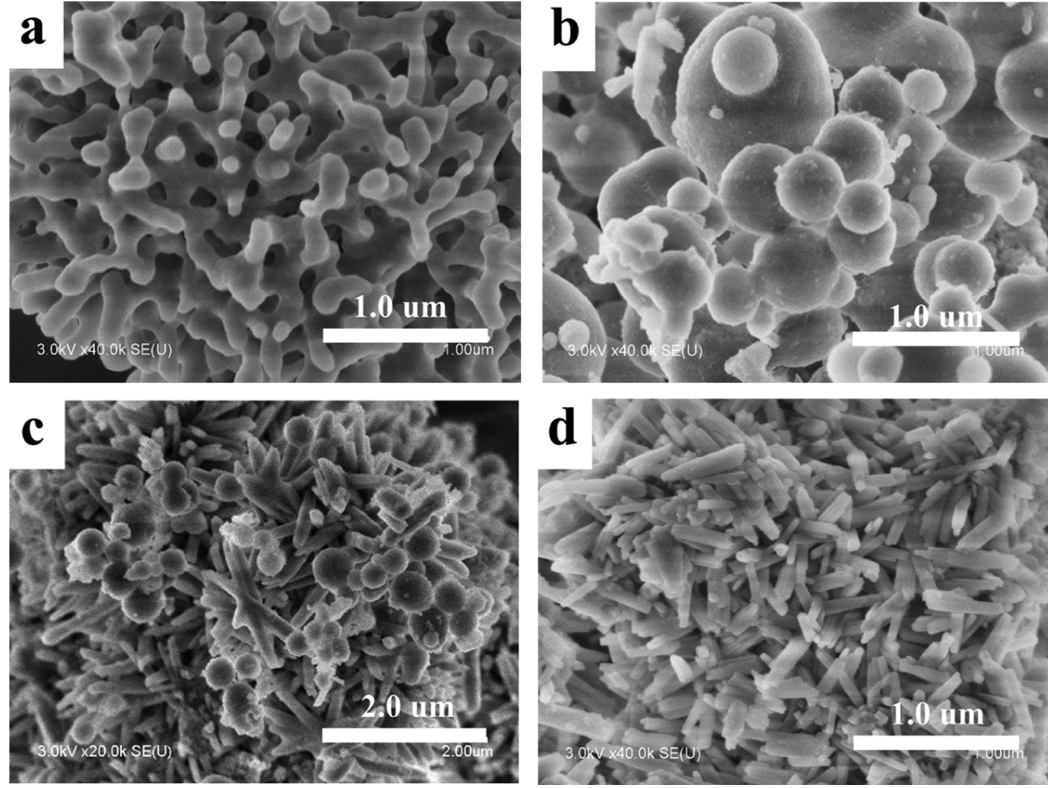

Figure 5. SEM images of samples collected at 6 (a), 12 (b), 36 (c), and 88 h (d).

Table 1. $\mathrm{SiO}_{2} / \mathrm{Al}_{2} \mathrm{O}_{3}$ Molar Ratios and Textural Properties of B-MTT and Al,B-MTT Samples

$\begin{array}{ccccccc}\text { no. }^{a} & \mathrm{SiO}_{2} / \mathrm{Al}_{2} \mathrm{O}_{3}{ }^{b} & S_{\text {BET }^{c}\left(\mathrm{~m}^{2} / \mathrm{g}\right)} & S_{\text {micro }}{ }^{c}\left(\mathrm{~m}^{2} / \mathrm{g}\right) & S_{\text {ext }}{ }^{c}\left(\mathrm{~m}^{2} / \mathrm{g}\right) & V_{\text {total }}{ }^{c}\left(\mathrm{~cm}^{3} / \mathrm{g}\right) & V_{\text {micro }}^{c}\left(\mathrm{~cm}^{3} / \mathrm{g}\right) \\ \text { Al,B-MTT (run 18) } & 96 & 224 & 204 & 20 & 0.22 & 0.087 \\ \text { Al,B-MTT (run 19) } & 148 & 276 & 244 & 32 & 0.29 & 0.103 \\ \text { Al,B-MTT (run 20) } & 206 & 228 & 206 & 22 & 0.24 \\ \text { B-MTT (run 7) } & \text { inf } & 157 & 140 & 17 & 0.19 & 0.089\end{array}$

${ }^{a}$ All samples were synthesized using the gels with molar compositions of OTMAC $/ \mathrm{SiO}_{2}=0.3, \mathrm{NaOH} / \mathrm{SiO}_{2}=0.6$, and $\mathrm{H}_{2} \mathrm{O} / \mathrm{SiO}_{2}=25$ and 5.0 wt $\%$ calcined B-SFE seeds at $150{ }^{\circ} \mathrm{C}$ for $88 \mathrm{~h}$. ${ }^{b}$ Determined by ICP-AES. ${ }^{c}$ Determined by BET and $t$-plot methods.

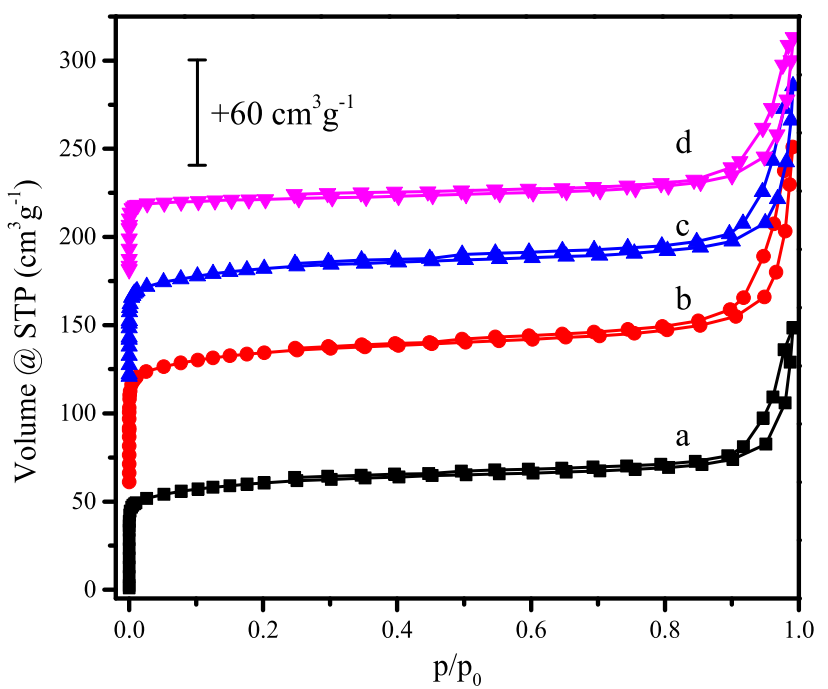

Figure 6. $\mathrm{N}_{2}$ adsorption-desorption isotherms of Al,B-MTT zeolites obtained with different $\mathrm{SiO}_{2} / \mathrm{Al}_{2} \mathrm{O}_{3}$ molar ratios: 200 (a), 400 (b), 560 (c), and B-MTT (d). The isotherms are offset vertically by 60 $\mathrm{cm}^{3} \mathrm{~g}^{-1}$ for clarity.

The incorporation of heteroatoms into the framework would endow zeolite with promising catalytic properties. ${ }^{33,45}$ Attempts to synthesize $\mathrm{Zr} / \mathrm{V} / \mathrm{Fe}$ incorporated B-MTT zeolites were carried out. The detailed synthetic parameters are listed in Table S3. XRD patterns confirmed the successful formation of the MTT structure (Figure S6). All samples show rodlike morphologies, similar to that of B-MTT zeolite (Figure S7).
The incorporation of heteroatoms was confirmed by the UVvisible spectra and UV Raman spectra shown in Figure 9A,B, respectively. For B-MTT, there is an extremely weak band at $211 \mathrm{~nm}$ in the UV-visible spectrum, which is typically attributed to the charge transfer from the framework oxygen $(\mathrm{Oz})$ to the other framework hybrid atoms ( $\mathrm{Si}$ or $\mathrm{B}$ ) of zeolite. ${ }^{46}$ The Raman spectrum of the B-MTT shows bands at 525,775 , and $1170 \mathrm{~cm}^{-1}$, which are assigned to the symmetric stretching and asymmetric stretching vibrations of framework $\mathrm{Si}-\mathrm{O}-\mathrm{Si}$ species due to the resonance Raman effect. ${ }^{47} \mathrm{Zr}, \mathrm{B}$ MTT zeolite exhibited a strong absorbance band at $212 \mathrm{~nm}$ and a weak absorbance band at $244 \mathrm{~nm}$ in the UV-visible spectrum ascribed to the charge-transfer transition between $\mathrm{O}^{2-}$ and the tetrahedral $\mathrm{Zr}^{2+}$. The bands at 490, 640, and 991 $\mathrm{cm}^{-1}$ in the Raman spectrum are generally accepted as the catalytically active $\mathrm{Zr}$ species with tetrahedral coordinations. ${ }^{48,49}$ For V,B-MTT, the two strong bands at 215 and $240 \mathrm{~nm}$ in the UV-visible spectrum and three bands at 931, 989 , and $1038 \mathrm{~cm}^{-1}$ in the Raman spectrum demonstrate the dominating existence of tetrahedral vanadium species in V,BMTT. The broad band at around $290 \mathrm{~nm}$ in the UV-visible spectrum reveals the formation of a small amount of vanadium nanoclusters. ${ }^{50,51}$ The Fe,B-MTT sample exhibits two very strong absorbance bands at 217 and $239 \mathrm{~nm}$ in the UV-visible spectrum, which are assigned to electron transfer from $\mathrm{O}^{2-}$ to tetrahedral $\mathrm{Fe}^{3+}$, a framework-doped ion. ${ }^{47}$ The Raman spectra of the samples show strong Raman bands at 520, 636, and $1040 \mathrm{~cm}^{-1}$, which have been assigned to the symmetric stretching and asymmetric stretching vibrations of framework $\mathrm{Fe}-\mathrm{O}-\mathrm{Si}$ species due to the resonance Raman effect. ${ }^{52,53}$ 

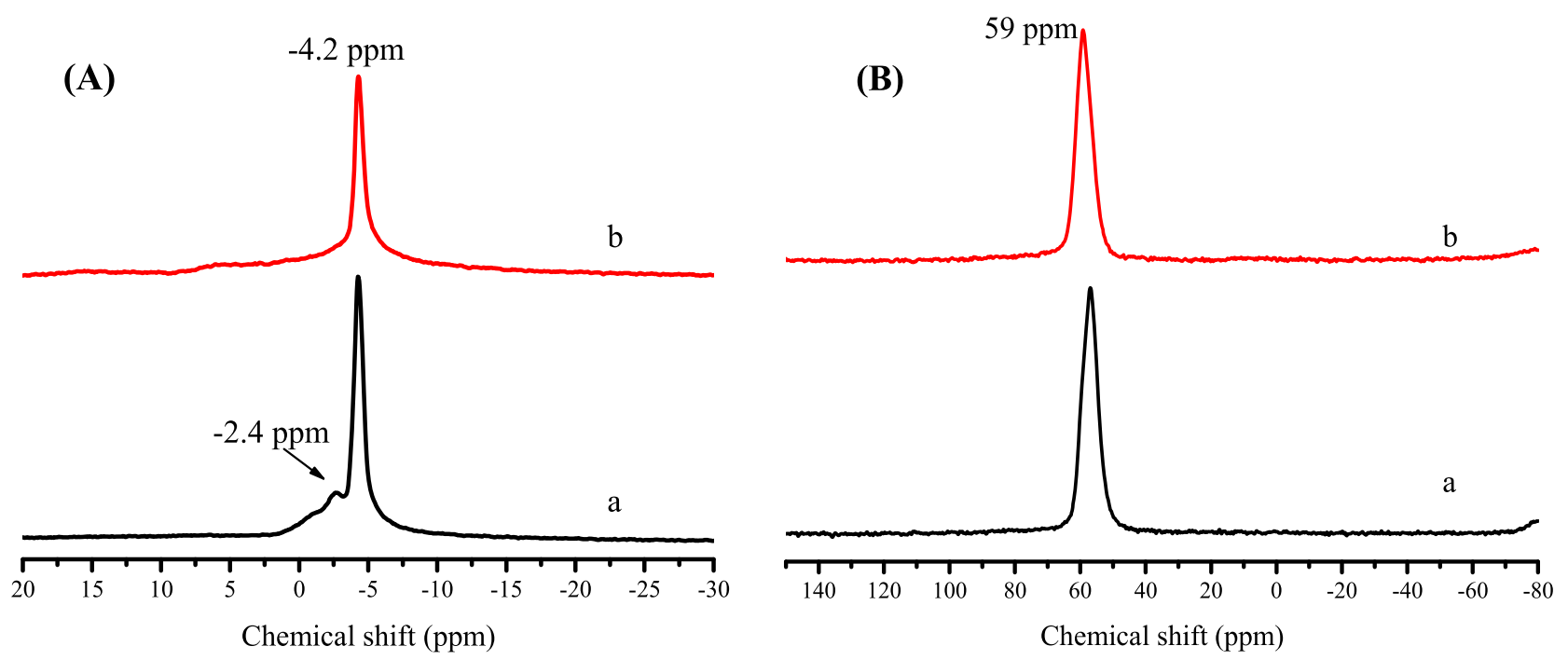

Figure 7. (A) Solid-state ${ }^{11} \mathrm{~B}$ MAS NMR spectra of the as-synthesized B-MTT, run 7 (a) and Al,B-MTT, run 18 (b); (B) solid-state ${ }^{27} \mathrm{Al}$ MAS NMR spectra of the as-synthesized Al,B-MTT-200, run 18 (a) and Al,B-MTT-560, run 20 (b). The numbers 200 and 560 represent $\mathrm{SiO}_{2} / \mathrm{Al}_{2} \mathrm{O}_{3}$ molar ratios of the gel.

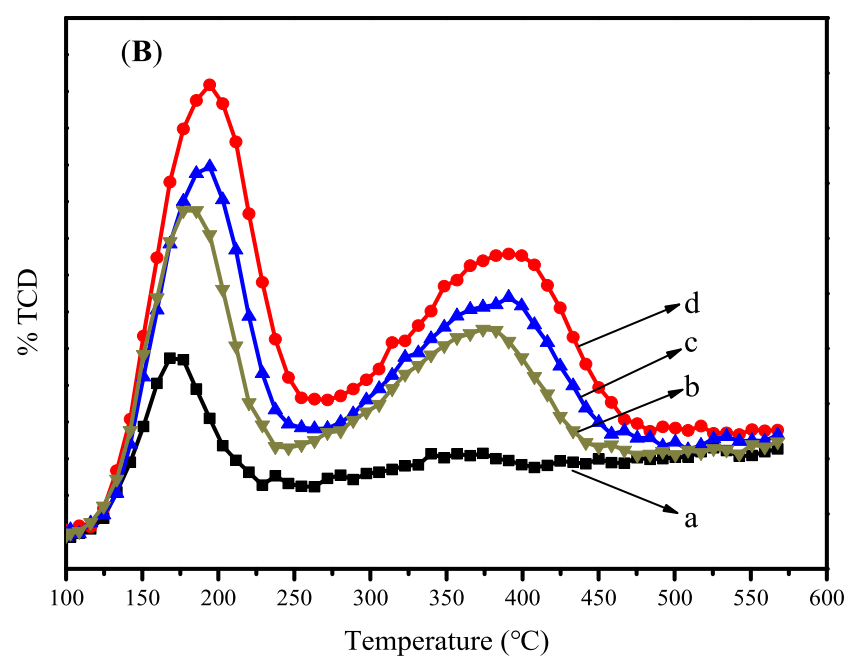

Figure 8. $\mathrm{NH}_{3}$-TPD curves of B-MTT, run 7 (a) and Al,B-MTT obtained from synthetic gels with different $\mathrm{SiO}_{2} / \mathrm{Al}_{2} \mathrm{O}_{3}$ molar ratios of 560, run 18 (b), 400, run 19 (c), and 200, run 20 (d).
These implied that $\mathrm{Fe}^{3+}$ ions are mainly located at isolated tetrahedral framework sites. It is expected that the incorporation of $\mathrm{Zr} / \mathrm{V} / \mathrm{Fe}$ would change the acidic properties and endow zeolite with different catalytic performances.

\section{CONCLUSIONS}

In summary, MTT-type zeolites, including B-MTT and Al,BMTT, and heteroatom-incorporated MTT zeolites were successfully synthesized in a borosilicate system with the presence of both SFE zeolite seed and OTMAC. The exotic SFE zeolite seed and OTMAC together act as the cooperative SDA and direct the formation of the MTT structure. The synthesis provides an example for further investigating the directing effects of zeolite seeds and OSDAs. The obtained Al,B-MTT and heteroatom-incorporated MTT zeolites may be promising materials for catalytic applications.

\section{EXPERIMENTAL SECTION}

4.1. Materials. Ludox ( 40 wt \%, HS-40 colloidal silica, Sigma-Aldrich), boric acid [99.8\% wt \%, China Medicine (Group)], sodium aluminate $\left[\mathrm{NaAlO}_{2}, \mathrm{Al}_{2} \mathrm{O}_{3} 40.4\right.$ wt \%, $\mathrm{Na}_{2} \mathrm{O}$
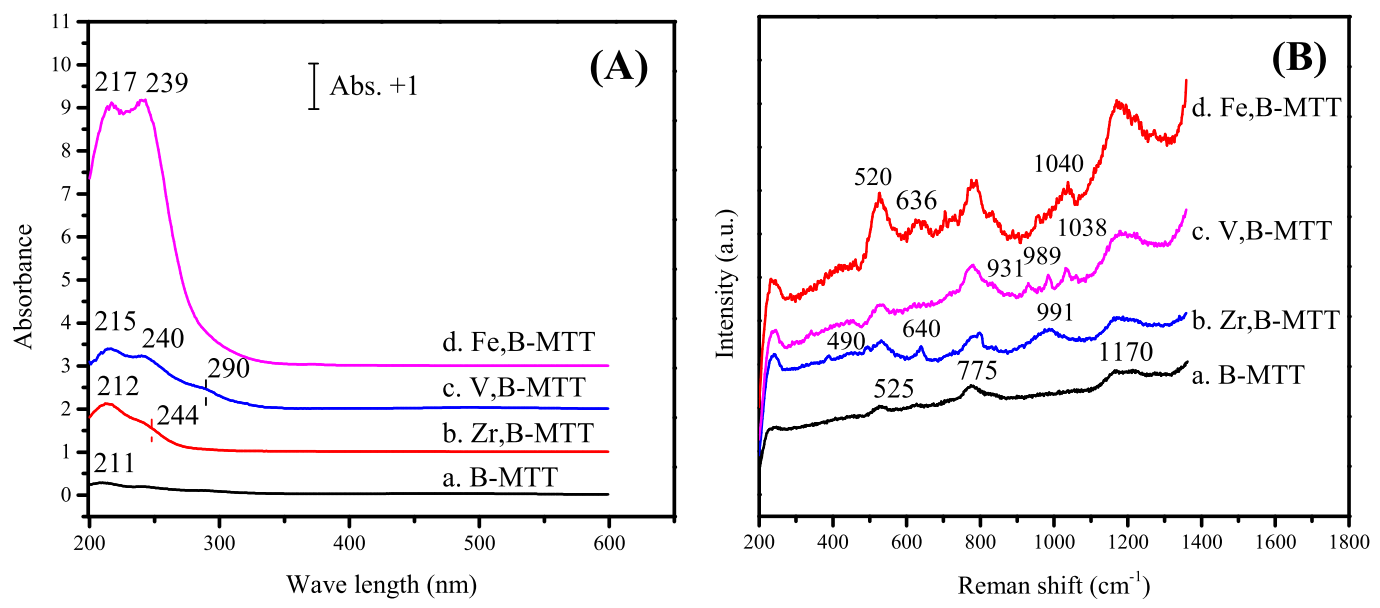

Figure 9. (A) UV-visible spectra and (B) UV Raman spectra of heteroatom zeolites with the MTT-type framework. 
30.6 wt \%, China Medicine (Group)], sodium hydroxide (NaOH, 96 wt \% Sinopharm Chemical Reagent Co. Ltd), 4dimethylaminopyridine [DMAP, $99.0 \mathrm{wt} \%$, TCI (Shanghai)], OTMAC [99.0 wt \%, TCI (Shanghai)], zirconium(IV) oxychloride octahydrate [99.0 wt \%, China Medicine (Group)], sodium metavanadate [99.0 wt \%, China Medicine (Group)], and iron(III) sulfate hydrate [Fe, $23.0 \mathrm{wt} \%$, China Medicine (Group)] were used as starting materials without any purifications. Deionized water was made in our laboratory.

4.2. Synthesis of Borosilicate SFE-type Zeolite Seed (B-SFE). B-SFE zeolite seed was prepared according to the literature ${ }^{39}$ with a reaction mixture with molar composition of $1 \mathrm{SiO}_{2}: 0.05 \quad \mathrm{~B}_{2} \mathrm{O}_{3}: 0.6$ DMAP:25 $\mathrm{H}_{2} \mathrm{O}$. The hydrothermal crystallization was carried out at $170{ }^{\circ} \mathrm{C}$ for $120 \mathrm{~h}$ under rotation $(20 \mathrm{rpm})$. The product was recovered by filtration, washed thoroughly with distilled water, and dried at $80{ }^{\circ} \mathrm{C}$ over night. The occluded OSDA was removed completely by calcination in an air atmosphere at $550{ }^{\circ} \mathrm{C}$ for $6 \mathrm{~h}$.

4.3. Syntheses of MTT-type Zeolites. Borosilicate MTTtype zeolite (B-MTT) was synthesized in the presence of OTMAC and B-SFE zeolite seed. Molar ratios of the starting mixtures and crystallization conditions are listed in Table S1. In a typical synthesis (run 7), $0.59 \mathrm{~g}$ of $\mathrm{NaOH}$ was dissolved in $8.87 \mathrm{~g}$ of deionized water, followed by the addition of $1.53 \mathrm{~g}$ of OTMAC and $0.31 \mathrm{~g}$ of boric acid. Then, $3.70 \mathrm{~g}$ of Ludox (40 wt \%) and $0.075 \mathrm{~g}$ of B-SFE zeolite seed (5.0 wt \% corresponding to $\mathrm{SiO}_{2}$ in the silica source) were added into the mixture to obtain a reaction gel with molar composition of $1.0 \mathrm{SiO}_{2}: 0.10 \quad \mathrm{~B}_{2} \mathrm{O}_{3}: 0.3$ OTMAC:0.6 NaOH:25 $\mathrm{H}_{2} \mathrm{O}$. After being stirred for $2 \mathrm{~h}$, a homogeneous gel was obtained and transferred to a stainless-steel autoclave. Hydrothermal crystallization was carried out at $150{ }^{\circ} \mathrm{C}$ for $88 \mathrm{~h}$. When the crystallization was over, the autoclave was quenched with cold water and the solid product was separated by centrifugation, washed with deionized water, dried at $80{ }^{\circ} \mathrm{C}$ for $12 \mathrm{~h}$, and calcined at $550{ }^{\circ} \mathrm{C}$ for $5 \mathrm{~h}$ in an air atmosphere.

The syntheses of $\mathrm{Al}$ and heteroatom-incorporated MTTtype zeolites (Al,B-MTT, Zr,B-MTT, V,B-MTT, anf Fe,BMTT) were synthesized following similar procedures for the synthesis of B-SFE zeolite except for the introduction of $\mathrm{NaAlO}_{2}$, zirconium(IV) oxychloride octahydrate, sodium metavanadate, and iron(III) sulfate hydrate as metal sources into the mixture before Ludox. The crystallizations of $\mathrm{Al} / \mathrm{Zr} /$ $\mathrm{V} / \mathrm{Fe}$-incorporated MTT-type zeolites were carried out under rotation at $150{ }^{\circ} \mathrm{C}$ for $96-168 \mathrm{~h}$.

The protonated forms of B-MTT and Al,B-MTT zeolites were obtained via ion exchange by adding $1.0 \mathrm{~g}$ of calcined zeolite into $25 \mathrm{~mL}$ of $0.5 \mathrm{M} \mathrm{NH}_{4} \mathrm{Cl}$ solution. The ion exchange was carried out three times under stirring at $80^{\circ} \mathrm{C}$ for $2 \mathrm{~h}$. The obtained samples were separated, dried, and calcined at $550^{\circ} \mathrm{C}$ for $5 \mathrm{~h}$. The obtained materials were denoted as H-B-MTT and $\mathrm{H}-\mathrm{Al}, \mathrm{B}-\mathrm{MTT}$, respectively.

4.4. Characterization. Powder XRD patterns were recorded on a D8 Advance SS X-ray diffractometer using $\mathrm{Cu}$ $\mathrm{K} \alpha$ radiation $(\lambda=1.54 \AA)$ operated at $40 \mathrm{kV}$ and $40 \mathrm{~mA}$. After subtracting the background using Origin software, the relative crystallinity of the MTT-type zeolite is calculated using the diffraction peak area in the $2 \theta$ range between 20 and $30^{\circ}$ in the XRD patterns. SEM measurements were performed on a Hitachi S-4800 field-emission scanning electron microscope (Hitachi, Japan) with an acceleration voltage of $3 \mathrm{kV}$. The transmission electron microscopy (TEM) image was collected on a JEOL JEM2100 TEM at $200 \mathrm{kV}$. Thermogravimetric analysis (TGA) was measured on a TA SDT-Q600 instrument by heating the samples up to $700{ }^{\circ} \mathrm{C}$ at a rate of $10{ }^{\circ} \mathrm{C} / \mathrm{min}$. Nitrogen adsorption isotherms were measured at $77 \mathrm{~K}$ on a Microtrac Belsorp-MaxII volumetric adsorption analyzer. The samples were outgassed at $573 \mathrm{~K}$ for $4 \mathrm{~h}$ before the measurements. The Brunauer-Emmet-Teller (BET) specific surface area was calculated based on the adsorption data acquired in a relative pressure $\left(p / p_{0}\right)$ range of $0.01-0.1$, and the total pore volume was determined from the amount adsorbed at a relative pressure of about 0.99 . The amounts of $\mathrm{Al}$ and $\mathrm{B}$ were quantified by inductively coupled plasma (ICP) on a Varian 725-ES instrument after dissolving the samples in $\mathrm{HF}$ solution. The liquid ${ }^{13} \mathrm{C}$ nuclear magnetic resonance spectrum was recorded on a Bruker AV-400 spectrometer. ${ }^{11} \mathrm{~B}$, ${ }^{27} \mathrm{Al}$, and ${ }^{13} \mathrm{C}$ magic angle spinning nuclear magnetic resonance (MAS NMR) measurements were performed using a Bruker 600 Ascent spectrometer. ${ }^{11} \mathrm{~B}$ and ${ }^{27} \mathrm{Al} \mathrm{NMR}$ spectra were recorded with a $4 \mathrm{~mm}$ T3HXY probe at $100.54 \mathrm{MHz}$ and a spinning rate of $9 \mathrm{kHz} .{ }^{13} \mathrm{C}$ NMR spectra were recorded with a $7.5 \mathrm{~mm}$ T3HX probe at $100.54 \mathrm{MHz}$ and a spinning rate of 5 $\mathrm{kHz}$. The acidity of the catalysts was measured by the temperature-programmed desorption of ammonia $\left(\mathrm{NH}_{3}-\right.$ TPD). UV Raman spectra were measured with a UV Raman-100 triple-stage spectrograph with a spectral resolution of $2 \mathrm{~cm}^{-1}$. The $244 \mathrm{~nm}$ line from a Coherent Innova 300 Fred laser with a power of around $1.0 \mathrm{~mW}$ on the sample was used. The accumulation time for the spectrum is $50 \mathrm{~s}$. UV-vis absorption spectra were measured at $258 \mathrm{C}$ using a VARIAN Cary5000 digital spectrophotometer equipped with an integrating sphere, in which $\mathrm{BaSO}_{4}$ was used as a reference.

\section{ASSOCIATED CONTENT}

\section{Supporting Information}

The Supporting Information is available free of charge at https://pubs.acs.org/doi/10.1021/acsomega.1c03810.

XRD patterns; SEM images; specific synthesis conditions; molar ratios and acidic concentrations; and textural properties of zeolite (PDF)

\section{AUTHOR INFORMATION}

\section{Corresponding Authors}

Zhendong Wang - State Key Laboratory of Green Chemical Engineering and Industrial Catalysis, Sinopec Shanghai Research Institute of Petrochemical Technology, Shanghai 201208, PR China; 이이이.org/0000-0003-1469-3912; Email: wangzd.sshy@sinopec.com

Weimin Yang - School of Chemical Engineering, East China University of Science and Technology, Shanghai 200237, PR China; State Key Laboratory of Green Chemical Engineering and Industrial Catalysis, Sinopec Shanghai Research Institute of Petrochemical Technology, Shanghai 201208, PR China; Email: yangwm.sshy@sinopec.com

\section{Authors}

Duozheng Ma - School of Chemical Engineering, East China University of Science and Technology, Shanghai 200237, PR China; State Key Laboratory of Green Chemical Engineering and Industrial Catalysis, Sinopec Shanghai Research Institute of Petrochemical Technology, Shanghai 201208, PR China

Wenhua Fu - State Key Laboratory of Green Chemical Engineering and Industrial Catalysis, Sinopec Shanghai 
Research Institute of Petrochemical Technology, Shanghai 201208, PR China; (i) orcid.org/0000-0001-6838-167X

Chuang Liu - State Key Laboratory of Green Chemical Engineering and Industrial Catalysis, Sinopec Shanghai Research Institute of Petrochemical Technology, Shanghai 201208, PR China

Jun Liang - School of Chemical Engineering, East China University of Science and Technology, Shanghai 200237, PR China; State Key Laboratory of Green Chemical Engineering and Industrial Catalysis, Sinopec Shanghai Research Institute of Petrochemical Technology, Shanghai 201208, PR China

Zhiqing Yuan - State Key Laboratory of Green Chemical Engineering and Industrial Catalysis, Sinopec Shanghai Research Institute of Petrochemical Technology, Shanghai 201208, PR China

Weichuan Tao - State Key Laboratory of Green Chemical Engineering and Industrial Catalysis, Sinopec Shanghai Research Institute of Petrochemical Technology, Shanghai 201208, PR China

Complete contact information is available at:

https://pubs.acs.org/10.1021/acsomega.1c03810

\section{Notes}

The authors declare no competing financial interest.

\section{ACKNOWLEDGMENTS}

This work was supported by the National Key R\&D Program of China (2017YFB0702800) and the National Natural Science Foundation of China (21972168 and 21802168).

\section{REFERENCES}

(1) Davis, M. E. Ordered porous materials for emerging applications. Nature 2002, 417, 813-821.

(2) Bandyopadhyay, R.; Kubota, Y.; Sugimoto, N.; Fukushima, Y.; Sugi, Y. Synthesis of borosilicate zeolites by the dry gel conversion method and their characterization. Microporous Mesoporous Mater. 1999, 32, 81-91.

(3) Li, J.; Corma, A.; Yu, J. Synthesis of new zeolite structures. Chem. Soc. Rev. 2015, 44, 7112-7127.

(4) Wagner, P.; Terasaki, O.; Ritsch, S.; Nery, J. G.; Zones, S. I.; Davis, M. E.; Hiraga, K. Electron Diffraction Structure Solution of a Nanocrystalline Zeolite at Atomic Resolution. J. Phys. Chem. B 1999, 103, 8245-8250.

(5) Wang, B.; Tian, Z.; Li, P.; Wang, L.; Xu, Y.; Qu, W.; He, Y.; Ma, H.; Xu, Z.; Lin, L. A novel approach to synthesize ZSM-23 zeolite involving N,N-dimethylformamide. Microporous Mesoporous Mater. 2010, 134, 203-209.

(6) Iyoki, K.; Takase, M.; Itabashi, K.; Muraoka, K.; Chaikittisilp, W.; Okubo, T. Organic structure-directing agent-free synthesis of NES-type zeolites using EU-1 seed crystals. Microporous Mesoporous Mater. 2015, 215, 191-198.

(7) Xu, Q.; Gong, Y.; Xu, W.; Xu, J.; Deng, F.; Dou, T. Synthesis of high-silica EU-1 zeolite in the presence of hexamethonium ions: A seeded approach for inhibiting ZSM-48. J. Colloid Interface Sci. 2011, $358,252-260$.

(8) Ji, Y.; Wang, Y.; Xie, B.; Xiao, F.-S. Zeolite Seeds: Third Type of Structure Directing Agents in the Synthesis of Zeolites. Comments Inorg. Chem. 2016, 36, 1-16.

(9) Majano, G.; Delmotte, L.; Valtchev, V.; Mintova, S. Al-Rich Zeolite Beta by Seeding in the Absence of Organic Template. Microporous Mesoporous Mater. 2009, 21, 4184-4191.

(10) Kamimura, Y.; Chaikittisilp, W.; Itabashi, K.; Shimojima, A.; Okubo, T. Critical factors in the seed-assisted synthesis of zeolite beta and "green beta" from OSDA-free $\mathrm{Na}$ +-aluminosilicate gels. Chem.Asian J. 2010, 5, 2182-2191.
(11) Kamimura, Y.; Iyoki, K.; Elangovan, S. P.; Itabashi, K.; Shimojima, A.; Okubo, T. OSDA-free synthesis of MTW-type zeolite from sodium aluminosilicate gels with zeolite beta seeds. Microporous Mesoporous Mater. 2012, 163, 282-290.

(12) Zhang, H.; Guo, Q.; Ren, L.; Yang, C.; Zhu, L.; Meng, X.; Li, C.; Xiao, F.-S. Organotemplate-free synthesis of high-silica ferrierite zeolite induced by CDO-structure zeolite building units. J. Mater. Chem. 2011, 21, 9494-9497.

(13) Zhang, H.; Yang, C.; Zhu, L.; Meng, X.; Yilmaz, B.; Müller, U.; Feyen, M.; Xiao, F.-S. Organotemplate-free and seed-directed synthesis of levyne zeolite. Microporous Mesoporous Mater. 2012, $155,1-7$.

(14) Ren, L.; Guo, Q.; Zhang, H.; Zhu, L.; Yang, C.; Wang, L.; Meng, X.; Feng, Z.; Li, C.; Xiao, F.-S. Organotemplate-free and onepot fabrication of nano-rod assembled plate-like micro-sized mordenite crystals. J. Mater. Chem. 2012, 22, 6564-6567.

(15) Iyoki, K.; Itabashi, K.; Okubo, T. Progress in seed-assisted synthesis of zeolites without using organic structure-directing agents. Microporous Mesoporous Mater. 2014, 189, 22-30.

(16) Itabashi, K.; Kamimura, Y.; Iyoki, K.; Shimojima, A.; Okubo, T. A Working Hypothesis for Broadening Framework Types of Zeolites in Seed-Assisted Synthesis without Organic Structure-Directing Agent. J. Am. Chem. Soc. 2012, 134, 11542-11549.

(17) Martín, N.; Paris, C.; Vennestrøm, P. N. R.; Thøgersen, J. R.; Moliner, M.; Corma, A. Cage-based small-pore catalysts for NH3SCR prepared by combining bulky organic structure directing agents with modified zeolites as reagents. Appl. Catal., B 2017, 217, 125136.

(18) Chiyoda, O.; Davis, M. E. Hydrothermal conversion of Yzeolite using alkaline-earth cations. Microporous Mesoporous Mater. 1999, 32, 257-264.

(19) Yamasaki, Y.; Tsunoji, N.; Takamitsu, Y.; Sadakane, M.; Sano, T. Synthesis of phosphorus-modified small-pore zeolites utilizing tetraalkyl phosphonium cations as both structure-directing and phosphorous modification agents. Microporous Mesoporous Mater. 2016, 223, 129-139.

(20) Oleksiak, M. D.; Muraoka, K.; Hsieh, M. F.; Conato, M. T.; Shimojima, A.; Okubo, T.; Chaikittisilp, W.; Rimer, J. D. OrganicFree Synthesis of a Highly Siliceous Faujasite Zeolite with Spatially Biased Q4(nAl) Si Speciation. Angew. Chem., Int. Ed. 2017, 56, 13366-13371.

(21) Lee, Y.-R.; Soe, J. T.; Zhang, S.; Ahn, J.-W.; Park, M. B.; Ahn, W.-S. Synthesis of nanoporous materials via recycling coal fly ash and other solid wastes: A mini review. Chem. Eng. J. 2017, 317, 821-843.

(22) Zhang, H.; Zhang, H.; Wang, P.; Zhao, Y.; Shi, Z.; Zhang, Y.; Tang, Y. Organic template-free synthesis of zeolite mordenite nanocrystals through exotic seed-assisted conversion. RSC Adv. 2016, 6, 47623-47631.

(23) Zhang, L.; Yang, C.; Meng, X.; Xie, B.; Wang, L.; Ren, L.; Ma, S.; Xiao, F.-S. Organotemplate-Free Syntheses of ZSM-34 Zeolite and Its Heteroatom-Substituted Analogues with Good Catalytic Performance. Chem. Mater. 2010, 22, 3099-3107.

(24) Nakazawa, N.; Ikeda, T.; Hiyoshi, N.; Yoshida, Y.; Han, Q.; Inagaki, S.; Kubota, Y. Correction to "A Microporous Aluminosilicate with 12-, 12-, and 8-Ring Pores and Isolated 8-Ring Channels". J. Am. Chem. Soc. 2017, 139, 12102.

(25) Fu, W.; Yuan, Z.; Jin, S.; Liu, W.; Wang, Z.; Wang, C.; Wang, Y.; Yang, W.; He, M.-Y. Cooperative structure-directing effect of choline cation and $* \mathrm{BEA}$ zeolite in the synthesis of aluminogermanosilicate IWR zeolite. Chin. J. Catal. 2019, 40, 856-866.

(26) Zhang, Z.; Qin, B.; Zhang, X.; Ling, F.; Sun, W.; Fang, X. The seeds effect on zeolite NU-87: synthesis parameters and structural properties. J. Porous Mater. 2013, 20, 515-521.

(27) Chen, Y.; Li, C.; Wang, L.; Zhang, M.; Liang, C. Seed-assisted synthesis of ZSM-23 zeolites in the absence of alkali metal ions. Microporous Mesoporous Mater. 2017, 252, 146-153.

(28) Liu, Y.; Wang, Z.; Ling, Y.; Li, X.; Liu, Y.; Wu, P. Synthesis of ZSM-23 Zeolite Using Isopropylamine as Template. Chin. J. Catal. 2009, 30, 525-530. 
(29) Molino, A.; Holzinger, J.; Łukaszuk, K. A.; Rojo-Gama, D.; Gunnæs, A. E.; Skibsted, J.; Lundegaard, L. F.; Svelle, S.; Beato, P.; Bordiga, S.; Lillerud, K. P. Synthesis of ZSM-23 (MTT) zeolites with different crystal morphology and intergrowths: effects on the catalytic performance in the conversion of methanol to hydrocarbons. Catal. Sci. Technol. 2019, 9, 6782-6792.

(30) Molino, A.; Łukaszuk, K. A.; Rojo-Gama, D.; Lillerud, K. P.; Olsbye, U.; Bordiga, S.; Svelle, S.; Beato, P. Conversion of methanol to hydrocarbons over zeolite ZSM-23 (MTT): exceptional effects of particle size on catalyst lifetime. Chem. Commun. 2017, 53, 68166819.

(31) Silva, B. J. B.; Sousa, L. V.; Sarmento, L. R. A.; Carvalho, R. P.; Quintela, P. H. L.; Pacheco, J. G. A.; Fréty, R.; Silva, A. O. S. Effect of desilication on the textural properties, acidity and catalytic activity of zeolite ZSM-23 synthesized with different structure-directing agents. Microporous Mesoporous Mater. 2019, 290, 109647.

(32) Chen, Y.; Li, C.; Chen, X.; Liu, Y.; Liang, C. Synthesis of ZSM23 zeolite with dual structure directing agents for hydroisomerization of n-hexadecane. Microporous Mesoporous Mater. 2018, 268, 216-224.

(33) Chen, Y.; Li, C.; Chen, X.; Liu, Y.; Tsang, C.-W.; Liang, C. Synthesis and Characterization of Iron-Substituted ZSM-23 Zeolite Catalysts with Highly Selective Hydroisomerization of n-Hexadecane. Ind. Eng. Chem. Res. 2018, 57, 13721-13730.

(34) Moini, A.; Schmitt, K. D.; Valyocsik, E. W.; Polomski, R. F. The role of diquaternary cations as directing agents in zeolite synthesis. Zeolites 1994, 14, 504-511.

(35) Yue, C.; Xie, W.; Liu, Y.; Wu, H.; Li, X.; Wu, P. Hydrothermal synthesis of MWW-type analogues using linear-type quaternary alkylammonium hydroxides as structure-directing agents. Microporous Mesoporous Mater. 2011, 142, 347-353.

(36) Wu, Q.; Wang, X.; Meng, X.; Yang, C.; Liu, Y.; Jin, Y.; Yang, Q.; Xiao, F.-S. Organotemplate-free, seed-directed, and rapid synthesis of Al-rich zeolite MTT with improved catalytic performance in isomerization of m-xylene. Microporous Mesoporous Mater. 2014, $186,106-112$.

(37) Zones, S. I.; Burton, A. W. Diquaternary structure-directing agents built upon charged imidazolium ring centers and their use in synthesis of one-dimensional pore zeolites. J. Mater. Chem. 2005, 15, 4215.

(38) Flanigen, E. M. A Review and New Perspectives in Zeolite Crystallization. In Molecular Sieves; Advances in Chemistry; American Chemical Society, 1973; Vol. 121, pp 119-139.

(39) Luo, Y.; Wang, Z.; Sun, J.; Wang, Y.; Jin, S.; Zhang, B.; Sun, H.; Yang, W. A Facile and Green Method for the Synthesis of SFE Borosilicate Zeolite and Its Heteroatom-Substituted Analogues with Promising Catalytic Performances. Chem.-Eur. J. 2018, 24, 306311.

(40) Fild, C.; Shantz, D. F.; Lobo, R. F.; Koller, H. Cation-induced transformation of boron-coordination in zeolites. Phys. Chem. Chem. Phys. 2000, 2, 3091-3098.

(41) Möller, K.; Bein, T. Crystallization and porosity of ZSM-23. Microporous Mesoporous Mater. 2011, 143, 253-262.

(42) Hwang, S.-J.; Chen, C.-Y.; Zones, S. I. Boron Sites in Borosilicate Zeolites at Various Stages of Hydration Studied by Solid State NMR Spectroscopy. J. Phys. Chem. B 2004, 108, 18535-18546.

(43) Zones, S. I.; Olmstead, M. M.; Santilli, D. S. Guest/host relationships in the synthesis of large pore zeolite SSZ-26 from a propellane quaternary ammonium compound. J. Am. Chem. Soc. 1992, 114, 4195-4201.

(44) Gabelica, Z.; Nagy, J. B.; Bodart, P.; Debras, G. High resolution solid state MAS 11B-NMR evidence of boron incorporation in tetrahedral sites of zeolites. Chem. Lett. 1984, 13, 1059-1062.

(45) Kumar, R.; Ratnasamy, P. Isomorphous substitution of iron in the framework of zeolite ZSM-23. J. Catal. 1990, 121, 89-98.

(46) Yang, K.; Wang, B.; Chen, L.; Wang, X. Synthesis and characterization of $\mathrm{Pd}-\mathrm{ZSM}-23$ with $\mathrm{Na}$ and $\mathrm{H}$ form for catalytic hydrodehalogenation of bromobenzene. Catal. Commun. 2008, 9, 431-436.
(47) Fan, F.; Sun, K.; Feng, Z.; Xia, H.; Han, B.; Lian, Y.; Ying, P.; Li, C. From Molecular Fragments to Crystals: A UV Raman Spectroscopic Study on the Mechanism of Fe-ZSM-5 Synthesis. Chem.-Eur. J. 2009, 15, 3268-3276.

(48) Ramanathan, A.; Castro Villalobos, M. C.; Kwakernaak, C.; Telalovic, S.; Hanefeld, U. Zr-TUD-1: a Lewis acidic, threedimensional, mesoporous, zirconium-containing catalyst. Chem.Eur. J. 2008, 14, 961-972.

(49) Tang, B.; Dai, W.; Sun, X.; Wu, G.; Guan, N.; Hunger, M.; Li, L. Mesoporous Zr-Beta zeolites prepared by a post-synthetic strategy as a robust Lewis acid catalyst for the ring-opening aminolysis of epoxides. Green Chem. 2015, 17, 1744-1755.

(50) Dzwigaj, S.; Matsuoka, M.; Anpo, M.; Che, M. Evidence of Three Kinds of Tetrahedral Vanadium (V) Species in VSi $\beta$ Zeolite by Diffuse Reflectance UV-Visible and Photoluminescence Spectroscopies. J. Phys. Chem. B 2000, 104, 6012-6020.

(51) Wu, Z.; Dai, S.; Overbury, S. H. Multiwavelength Raman Spectroscopic Study of Silica-Supported Vanadium Oxide Catalysts. J. Phys. Chem. C 2010, 114, 412-422.

(52) Guo, Q.; Fan, F.; Guo, M.; Feng, Z.; Li, C. UV Raman Spectroscopic Studies on the Synthesis Mechanism of FeAlPO4-5. Chin. J. Catal. 2012, 33, 106-113.

(53) Lewis, D. W.; Catlow, C. R. A.; Sankar, G.; Carr, S. W. Structure of Iron-Substituted ZSM-5. J. Phys. Chem. 1995, 99, 23772383. 